\title{
Hemogram, Fibrinogen Concentration and Plasma Total Protein from Calves of Criollo Lageano Breed Variety Hornless and Aberdeen Angus Breed (Red Angus) on the First Six Months of Life
}

\author{
Jackson Schade', Renato May Rossi', Mere Erika Saito', \\ Graziela Vieira Fonteque ${ }^{2}$, Thiago Rinaldi Muller' \& Joandes Henrique Fonteque ${ }^{1}$
}

\begin{abstract}
Background: Neonatal period in bovine presents important variations in hematologic and immunologic parameters, and the resulting mortality rates may be due to increased susceptibility to disease. Race, age, environmental, climate and management factors are sources of CBC's parameters variation in cattle. The objective of this study is to evaluate and compare the $\mathrm{CBC}$ variables and concentrations of total protein (PTP) and plasma fibrinogen calves of Criolo Lageano Breed variety hornless and Aberdeen Angus (Red Angus) in the first six months of life, which are produced under extensive management at the Planalto Serrano Catarinense (southern Brazil).

Materials, Methods \& Results: Two groups were selected, comprising 11 calves of Hornless Criolo Lageano and 14 calves of the Aberdeen Angus breed (Red Angus). Blood samples were collected for complete blood count and determination of total protein concentrations, and plasma fibrinogen in the periods between $24 \mathrm{~h}$ and 36, 15, 30, 60, 90, 120, 150 and 180 days of life. The statistical analysis was performed using analysis of variance for repeated measures (ANOVA) and Tukey's test $(P<0.05)$. During the experimental period infestations by ticks in both races were observed. In the first month of life, blood smears revealed the presence of blood parasites of the genus Babesia spp. and Anaplasma sp. The fluctuations seen for erythrocyte in the neonatal period (30 days), although not significant, consisted of raising the number of values of erythrocytes, hemoglobin $(\mathrm{Hb})$ and packed cell volume $(\mathrm{PCV})$, and reduction in mean corpuscular volume values (MCV). From 90 days of age there was a significant reduction in number of red blood cells and VG values, and elevated MCV values and mean corpuscular volume (MCHC) for the Aberdeen Angus (Red Angus). For the WBC, the neonatal period was marked by increase in the number of lymphocytes and decrease in neutrophil ratio: lymphocyte $(\mathrm{N}, \mathrm{L})$ with respect to the inversion of 15 days. In the age group 90 days of life was evident increase in the number of leukocytes and lymphocytes in both races. The plasma fibrinogen concentrations showed no significant variations during the trial period. The plasma total protein (PTP), had higher values on the second day of life, with reductions up to 60 days and gradual increase in subsequent times.

Discussion: The fluctuations related to the values of erythrocyte variables in the neonatal period are consistent with results reported by other authors in calves of other races. Changes in erythrocyte values observed from 90 days of age on race Aberdeen Angus (Red Angus) can be attributed to infection by blood parasites and consequent hemolysis. As for the leucocyte count, increasing the number of lymphocytes and inversion of $\mathrm{N}: \mathrm{L}$ is the neonatal period in a physiological variation and confirms observations made by other authors in calves of other races. In the age group 90 days, leukocytosis by lymphocytosis was related to natural premonition against Babesia spp. and Anaplasma spp., which is documented in cattle in tropical and subtropical regions. When analyzing the PTP values on the second day of life, it can be said that there has been proper transfer of passive immunity in both races, given the high correlation of PTP values and gamma globulins. The reduction of PTP and subsequent elevation values are characteristic of the physiological curve related to degradation of passively transferred immunoglobulin and immunoglobulin synthesis by competent immune system.
\end{abstract}

Keywords: bovine, erythrogram, leukogram, fibrinogen, total protein.

Descritores: bovino, eritrograma, fibrinogênio, leucograma, proteína total. 


\section{INTRODUÇÃO}

A mortalidade de bezerros nos primeiros meses de vida representa perdas econômicas consideráveis para a bovinocultura mundial $[2,13,10,16]$. O período neonatal é marcado por rápidas e intensas modificações do quadro hematológico, caracterizando-se ainda, por maior susceptibilidade às doenças [20-22]. Nesse contexto, a hematologia assume fundamental importância, especialmente nos diagnósticos e prognósticos das enfermidades [23].

Estudos voltados aos constituintes sanguíneos de bovinos, incluindo o hemograma, são de extrema importância para avaliação das condições orgânicas, orientação na conduta terapêutica e evolução da doença [14]. Na espécie bovina, diversos são os fatores que influenciam variações no hemograma, tais como os fatores etários, sexuais, raciais e nutricionais, além de condições ambientais, climáticas e de manejo [5,23].

São inexistentes os trabalhos referentes à avaliação hematológica em bezerros das raças Crioula Lageana variedade mocha e Aberdeen Angus (Red Angus). Sendo assim, o objetivo do presente trabalho foi avaliar e comparar as variáveis do hemograma e as concentrações de proteína total e fibrinogênio plasmático de bezerros das raças Crioula Lageana variedade mocha e Aberdeen Angus (Red Angus), nos primeiros seis meses de vida, criados sob manejo extensivo no Planalto Serrano Catarinense.

\section{MATERIAIS E MÉTODOS}

\section{Delineamento experimental}

A realização do projeto envolveu uma equipe multidisciplinar e interinstitucional composta pela Universidade do Estado de Santa Catarina (UDESC), Centro Universitário Unifacvest e a Associação Brasileira de Criadores de Bovinos da Raça Crioula Lageana (ABCCL). O delineamento experimental foi inteiramente ao acaso. Foram selecionados aleatoriamente 25 bezerros aparentemente sadios ao nascimento e no primeiro dia de vida, produtos de partos eutócicos, os quais foram mantidos sob o mesmo manejo extensivo durante o período experimental. Os animais eram oriundos de um Núcleo de Conservação in situ da raça Crioula Lageana e Aberdeen Angus localizado na Fazenda Bom Jesus do Herval no município de Ponte Alta, SC, Brasil. Os bezerros foram divididos em dois grupos experimentais de acordo com a raça, compos- tos por 11 animais da raça Crioula Lageana variedade mocha e 14 da raça Aberdeen Angus (Red Angus).

\section{Obtenção das amostras}

Amostras de sangue foram colhidas de cada bezerro por venopunção jugular externa nas idades entre 24 e 36 h, 15, 30, 60, 90, 120, 150 e 180 dias de vida, em sistema à vácuo, por meio da utilização de agulhas 25 x 0,8 mm (Precision Glide) ${ }^{1}$ e frascos com anticoagulante EDTA tripotássico (Hemogard K3 Vacutainer Systems) ${ }^{1}$.

\section{Avaliação hematológica}

Para as contagens de eritrócitos e leucócitos totais utilizou-se a técnica hemocitométrica em câmara de Neubauer, sendo a contagem diferencial de leucócitos realizada por meio da análise microscópica de esfregaços sanguíneos corados com corantes do tipo Romanowsky (Panótico Rápido) ${ }^{2}$ [14]. O volume globular (VG) foi determinado pela técnica do microhematócrito e a concentração de hemoglobina $(\mathrm{Hb})$ foi analisada pelo método colorimétrico de acordo com a técnica da cianohemoglobina [14]. Após a obtenção dos valores de número de eritrócitos, concentração de hemoglobina e VG, foram calculados índices hematimétricos absolutos que consistiram em: Volume Corpuscular Médio (VCM), Hemoglobina Corpuscular Média (HCM) e Concentração de Hemoglobina Corpuscular Média (CHCM) [14]. A concentração da proteína total plasmática (PTP) foi determinada por refratometria (ATAGO) ${ }^{3}$ e a concentração do fibrinogênio plasmático pelo método de precipitação pelo calor seguida por leitura em refratômetro3 [14].

\section{Análise estatística}

A análise estatística dos dados realizada por meio Análise de Variância de Medidas Repetidas (ANOVA) e teste de Tukey, admitindo-se uma probabilidade de erro de $5 \%$ [9].

\section{RESULTADOS}

Durante o período experimental foram observadas infestações por carrapatos [Rhipicephalus (Boophilus) microplus], em ambas as raças, a partir do primeiro mês de vida. Foram evidenciadas maiores alterações clínicas na raça Aberdeen Angus (Red Angus), incluindo a infestação por carrapatos, sendo que um dos animais apresentou quadro clínico de tristeza parasitária bovina aos 150 dias e foi tratado 
com dipropionato de imidocarb $\left(\operatorname{Imizol}^{\circledR}\right)^{4}$. Produtos à base de ivermectina ou cipermetrina foram utilizados, de acordo com a necessidade, para o controle de infestações moderadas a graves.

Os valores médios e desvios-padrão referentes às variáveis do eritrograma de bezerros das raças Crioula Lageana variedade mocha e Aberdeen Angus (Red Angus) estão expressos na Tabela 1. Quando avaliado o período neonatal (até 30 dias de vida), os valores referentes às variáveis número de eritrócitos, $\mathrm{Hb}$ e VG, apesar de não significativos $(P>0,05)$, evoluíram com tendência ao aumento, com a progressão da idade, a partir do segundo dia (M1) até os 30 dias de vida (M3) para a raça Aberdeen Angus (Red Angus). Resultado semelhante foi observado para a raça Crioula Lageana, no entanto, a variável $\mathrm{Hg}$ apresentou diminuição aos 15 dias (M2), seguido por aumento aos 30 dias de idade (M3). Apesar de não diferir $(P>0,05)$ entre as faixas etárias, para ambas as raças, houve evolução com tendência a diminuição dos valores do VCM a partir do segundo dia (M1) até os 30 dias de vida (M3). Quanto a variável HCM houve tendência a diminuição dos valores $(P>0,05)$ a partir do segundo dia (M1) até os 30 dias de vida (M3) para a raça Aberdeen Angus (Red Angus). Na raça Crioula Lageana variedade mocha os valores de HCM tenderam a diminuir até os 15 dias, para aumentarem aos 30 dias de idade (M3) $[P>0,05]$.

Não houve diferença $(P>0,05)$ para as variáveis do eritrograma, de acordo com a faixa etária, nos bezerros da raça Crioula Lageana variedade mocha (Tabela 1). No entanto, para a raça Aberdeen Angus (Red Angus) foi observada diminuição nos valores médios de número de eritrócitos e VG, além de aumento dos valores de VGM e CHCM a partir dos 90 dias de idade (M5) $[P<0,05]$. Durante a análise dos esfregaços sanguíneos foi observada presença de hemoparasitos do gênero Babesia spp. e Anaplasma sp., em grande parte dos animais, em ambas as raças.

Tabela 1. Valores médios e desvios-padrão $(\mathrm{x} \pm \mathrm{s})$ do número de eritrócitos (Eritro), concentração de hemoglobina (Hemog), Volume globular (VG), Volume corpuscular médio (VCM), Hemoglobina corpuscular média (HCM) e Concentração de hemoglobina corpuscular média (CHCM) de bezerros das raças Crioula Lageana variedade Mocha e Aberdeen Angus (Red Angus), às 24-36 h (M1), 15 (M2), 30 (M3), 60 (M4), 90 (M5), 120 (M6), 150 (M7) e 180 dias (M8) de vida.

\begin{tabular}{|c|c|c|c|c|c|c|c|c|}
\hline Mon & M1 & M2 & M3 & M4 & M5 & M6 & M7 & M8 \\
\hline \multicolumn{9}{|c|}{ Crioula Lageana variedade Mocha } \\
\hline Eritro $\left(10^{6} / \mu \mathrm{L}\right)$ & $7,63 \pm 1,74^{\mathrm{Aa}}$ & $7,95 \pm 1,05^{\mathrm{Aa}}$ & $8,38 \pm 1,33^{\mathrm{Aa}}$ & $8,75 \pm 1,28^{\mathrm{Aa}}$ & $8,00 \pm 2,02^{\mathrm{Aa}}$ & $7,82 \pm 2,11^{\mathrm{Aa}}$ & $7,63 \pm 1,74^{\mathrm{Aa}}$ & $7,76 \pm 1,73^{\mathrm{Aa}}$ \\
\hline $\operatorname{Hemog}(\mathrm{g} / \mathrm{dL})$ & $11,84 \pm 2,70^{\mathrm{Aa}}$ & $11,58 \pm 1,96^{\mathrm{Aa}}$ & $12,24 \pm 1,94^{\mathrm{Aa}}$ & $12,59 \pm 2,24^{\mathrm{Aa}}$ & $13,02 \pm 2,13^{\mathrm{Aa}}$ & $11,68 \pm 1,87^{\mathrm{Aa}}$ & $11,39 \pm 1,99^{\mathrm{Aa}}$ & $11,73 \pm 1,69^{\mathrm{Aa}}$ \\
\hline $\mathrm{VG}(\%)$ & $36,27 \pm 7,44^{\mathrm{Aa}}$ & $36,20 \pm 5,05^{\mathrm{Aa}}$ & $38,60 \pm 3,84^{\mathrm{Aa}}$ & $38,10 \pm 4,95^{\mathrm{Aa}}$ & $37,80 \pm 5,75^{\mathrm{Aa}}$ & $34,90 \pm 4,82^{\mathrm{Aa}}$ & $33,50 \pm 5,28^{\mathrm{Aa}}$ & $35,00 \pm 5,19^{\mathrm{Aa}}$ \\
\hline VCM (fL) & $48,01 \pm 4,47^{\text {Аа }}$ & $45,62 \pm 3,48^{\mathrm{Aa}}$ & $46,58 \pm 4,52^{\mathrm{Aa}}$ & $43,90 \pm 4,67^{\mathrm{Aa}}$ & $48,33 \pm 6,11^{\text {Aa }}$ & $48,00 \pm 14,53^{\text {Aa }}$ & $44,92 \pm 6,11^{\mathrm{Aa}}$ & $45,78 \pm 4,26^{\mathrm{Aa}}$ \\
\hline $\mathrm{HCM}(\mathrm{pg})$ & $15,72 \pm 1,78^{\mathrm{Aa}}$ & $14,65 \pm 2,13^{\mathrm{Aa}}$ & $14,80 \pm 2,41^{\mathrm{Aa}}$ & $14,58 \pm 3,14^{\mathrm{Aa}}$ & $16,74 \pm 2,69^{\mathrm{Aa}}$ & $15,89 \pm 4,19^{\mathrm{Aa}}$ & $15,21 \pm 1,91^{\mathrm{Aa}}$ & $15,37 \pm 1,70^{\mathrm{Aa}}$ \\
\hline CHCM (g/dL) & $32,38 \pm 1,94^{\mathrm{Aa}}$ & $32,03 \pm 3,39^{\mathrm{Aa}}$ & $31,66 \pm 3,13^{\mathrm{Aa}}$ & $32,99 \pm 4,31^{\mathrm{Aa}}$ & $34,58 \pm 3,62^{\mathrm{Aa}}$ & $34,59 \pm 4,44^{\mathrm{Aa}}$ & $33,99 \pm 2,41^{\mathrm{Aa}}$ & $33,56 \pm 1,79^{\mathrm{Aa}}$ \\
\hline \multicolumn{9}{|c|}{ Aberdeen Angus (Red Angus) } \\
\hline Eritro $\left(10^{6} / \mu \mathrm{L}\right)$ & $7,40 \pm 1,53^{\mathrm{Aac}}$ & $8,42 \pm 0,84^{\mathrm{Aa}}$ & $8,58 \pm 1,24^{\mathrm{Aa}}$ & $8,61 \pm 1,46^{\mathrm{Aa}}$ & $7,77 \pm 1,57^{\mathrm{Aac}}$ & $6,99 \pm 1,76^{\mathrm{Aac}}$ & $6,31 \pm 1,91^{\mathrm{Abc}}$ & $6,13 \pm 1,29^{\mathrm{Bbc}}$ \\
\hline Hemog $(g / d L)$ & $11,10 \pm 1,47^{\mathrm{Aa}}$ & $12,17 \pm 1,23^{\mathrm{Aa}}$ & $12,49 \pm 1,14^{\mathrm{Aa}}$ & $11,29 \pm 2,06^{\mathrm{Aa}}$ & $10,59 \pm 1,57^{\mathrm{Ba}}$ & $10,88 \pm 1,64^{\mathrm{Aa}}$ & $10,05 \pm 1,80^{\mathrm{Aa}}$ & $9,97 \pm 1,19^{\mathrm{Ba}}$ \\
\hline $\mathrm{VG}(\%)$ & $33,33 \pm 4,42^{\mathrm{Aac}}$ & $37,83 \pm 3,76^{\mathrm{Aa}}$ & $37,92 \pm 3,78^{\mathrm{Aa}}$ & $34,92 \pm 5,55^{\mathrm{Aac}}$ & $31,67 \pm 4,31^{\mathrm{Bbc}}$ & $31,42 \pm 4,38^{\mathrm{Abc}}$ & $28,75 \pm 4,92^{\mathrm{Bbc}}$ & $29,42 \pm 3,73^{\mathrm{Bbc}}$ \\
\hline VCM (fL) & $47,02 \pm 6,13^{\mathrm{Aac}}$ & $45,16 \pm 4,98^{\mathrm{Aac}}$ & $44,52 \pm 4,40^{\text {Aac }}$ & $40,91 \pm 3,91^{\mathrm{Abc}}$ & $41,47 \pm 4,97^{\mathrm{Bbc}}$ & $45,56 \pm 6,80^{\mathrm{Aac}}$ & $47,83 \pm 9,01^{\mathrm{Aac}}$ & $49,17 \pm 7,80^{\mathrm{Aa}}$ \\
\hline $\mathrm{HCM}(\mathrm{pg})$ & $14,87 \pm 2,36^{\mathrm{Aac}}$ & $14,48 \pm 0,81^{\mathrm{Aac}}$ & $13,85 \pm 3,71^{\mathrm{Aac}}$ & $13,19 \pm 1,63^{\mathrm{Abc}}$ & $13,90 \pm 2,05^{\mathrm{Bac}}$ & $16,05 \pm 2,69^{\text {Aac }}$ & $16,76 \pm 3,58^{\mathrm{Aa}}$ & $16,74 \pm 3,03^{\mathrm{Aa}}$ \\
\hline $\mathrm{CHCM}(\mathrm{g} / \mathrm{dL})$ & $32,32 \pm 1,25^{\mathrm{Abc}}$ & $32,25 \pm 2,39^{\mathrm{Abc}}$ & $32,95 \pm 1,39^{\text {Aac }}$ & $32,30 \pm 2,42^{\mathrm{Abc}}$ & $33,46 \pm 2,53^{\mathrm{Aac}}$ & $34,65 \pm 2,28^{\mathrm{Aac}}$ & $34,93 \pm 1,98^{\mathrm{Aa}}$ & $33,97 \pm 1,95^{\text {Aac }}$ \\
\hline
\end{tabular}

APara letras maiúsculas iguais não há diferença significativa $(P>0,05)$ entre os grupos. aPara letras minúsculas iguais não há diferença significativa $(P>0,05)$ entre os momentos.

Com referência às variáveis do leucograma (Tabela 2), observou-se elevação $(P<0,05)$ dos valores médios referentes a contagem total de leucócitos e linfócitos na faixa etária dos 90 dias (M5) até 180 dias (M8) em ambas as raças. O número de neutrófilos bastonetes foi maior $(P<0,05)$ entre 24 e 36 h (M1) em relação aos momentos subsequentes em ambas às raças. Ocorreu aumento do número absoluto de linfócitos, bem como diminuição da relação neutrófilo:linfócito (N:L) após o nascimento e com o avançar da idade em ambas as raças, sendo a inversão da relação observada aos 15 dias (M2). Houve aumento $(P<0,05)$ dos valores médios de eosinófilos aos 120 dias de idade (M6) e maiores valores de neutrófilos bastonetes em ambas as raças, e basófilos $(P<0,05)$ para a raça Aberdeen Angus (Red Angus) no segundo dia de vida (M1). 
A análise da concentração do fibrinogênio plasmático (Tabela 2) não demonstrou diferença $(P>$ $0,05)$ de acordo com a faixa etária. No entanto, houve diferença quando comparadas as raças unicamente aos 120 dias de idade (M6), observando-se valores superiores $(P<0,05)$ para a raça Crioula Lageana variedade mocha.

Quanto a concentração de PTP (Tabela 2), seus valores médios foram superiores $(P<0,05)$ no momento compreendido entre 24 e $36 \mathrm{~h}$ de vida (M1), com redução de grande magnitude $(P<0,05)$ até os 15 dias de idade (M2), seguido por posterior redução gradativa até alcançar valores mínimos aos 60 dias de idade (M4) em ambas as raças. A partir dos 60 dias (M4) houve aumento gradativo dos valores de PPT em ambas as raças. Ao comparar-se os valores médios referentes a PTP entre as raças, não foi observada diferença $(P>0,05)$ no segundo dia de vida (24-36 h). No entanto aos 30 (M3) e 60 (M4) dias de vida houve diferença entre raças $(P<0,05)$, quando valores superiores foram observados para a raça Crioula Lageana variedade mocha.

Tabela 2. Valores médios e desvios-padrão $(\mathrm{x} \pm \mathrm{s}$ ) da contagem total (Leuco) e diferencial de leucócitos: Neutrófilos bastonetes (Bast), Neutrófilos segmentados (Seg), Linfócitos (Linf), Monócitos (Mono), Eosinófilos (Eosi), Basófilos (Baso), relação neutrófilo:linfócito (N:L), concentração de Fibrinogênio plasmático (Fib) e concentração de proteínas totais plasmáticas (PTP) de bezerros das raças Crioula Lageana variedade Mocha e Aberdeen Angus (Red Angus) às 24-36 h (M1), 15 (M2), 30 (M3), 60 (M4), 90 (M5), 120 (M6), 150 (M7) e 180 dias (M8) de vida.

\begin{tabular}{|c|c|c|c|c|c|c|c|c|}
\hline & & 12 & 43 & M4 & 15 & M6 & M7 & M8 \\
\hline \multicolumn{9}{|c|}{ Crioula Lageana variedade Mocha } \\
\hline Leuco $(x$ & $9,13 \pm 4,13^{\mathrm{Ab}}$ & $7,12 \pm 2,01^{\mathrm{Ab}}$ & $7,83 \pm 2,55^{\mathrm{Ab}}$ & $0,70 \pm 3,83^{\mathrm{Abc}}$ & $13,56 \pm 3,72^{\text {Aac }}$ & $13,91 \pm 4,62^{\text {Aac }}$ & $16,26 \pm 3,82^{\mathrm{Aa}}$ & $13,46 \pm 2,54^{\text {Aac }}$ \\
\hline Bast & $014+4$ & $0,02 \pm 0,05^{\mathrm{Ab}}$ & $0,00 \pm 0,00^{\mathrm{Ab}}$ & $0,00 \pm 0,00^{\mathrm{Ab}}$ & $0,00 \pm 0,00^{\mathrm{Ab}}$ & & $0,00 \pm 0,00^{\mathrm{Ab}}$ & \\
\hline Seg $(x$ & $6,57 \pm 4,15^{\text {Аa }}$ & $2,74 \pm 1,26^{\mathrm{Ab}}$ & $2,97 \pm 1,59^{\mathrm{Ab}}$ & $2,32 \pm 1,15^{\mathrm{Ab}}$ & $2,58 \pm 1,64^{\mathrm{Ab}}$ & $215+133 \mathrm{Ab}$ & $2,05 \pm 1,10^{\mathrm{Ab}}$ & $2,47 \pm 1,51^{\mathrm{Ab}}$ \\
\hline $\operatorname{Linf}($ & $2 \Omega 4 \Omega 40 \mathrm{ab}$ & $4,34 \pm 1$ & $4,61 \pm 1,32^{\mathrm{Ab}}$ & $8,06 \pm 2,86^{\mathrm{Ac}}$ & $10,53 \pm 3,00^{\mathrm{Ac}}$ & $11,45 \pm 4,48^{\mathrm{Ac}}$ & $13,65 \pm 3,51^{\mathrm{Aa}}$ & $10,49 \pm 2,06^{\mathrm{Ac}}$ \\
\hline Mono $\left(\times 10^{3} / \mu \mathrm{L}\right)$ & $0,13 \pm 0,11^{\mathrm{Aa}}$ & $0,12 \pm 0,09^{\mathrm{Aa}}$ & $0,26 \pm 0,43^{\mathrm{Aa}}$ & $0,26 \pm 0,49^{\mathrm{Aa}}$ & $0,22 \pm 0,20^{\mathrm{Aa}}$ & $0,16 \pm 0,11^{\mathrm{Ba}}$ & $0,44 \pm 0,45^{\mathrm{Aa}}$ & $0,34 \pm 0,46^{\mathrm{Aa}}$ \\
\hline $\operatorname{Eosi}\left(\mathrm{x} 10^{3} / \mu \mathrm{L}\right)$ & $0,04 \pm 0,06^{\mathrm{Aa}}$ & $0,03 \pm 0,05^{\text {Aa }}$ & $0,02 \pm 0,03^{\mathrm{Aa}}$ & $0,07 \pm 0,13^{\mathrm{Aa}}$ & $0,10 \pm 0,11^{\mathrm{Aa}}$ & $0,16 \pm 0,21^{\text {Aa }}$ & $0,11 \pm 0,22^{\mathrm{Aa}}$ & $0,15 \pm 0,17^{\mathrm{Aa}}$ \\
\hline Baso $\left(x 10^{3} / \mu \mathrm{L}\right)$ & $0,01 \pm 0,04^{\mathrm{Aa}}$ & $0,00 \pm 0,00^{\mathrm{Aa}}$ & $0,00 \pm 0,00^{\wedge a}$ & & $0,14 \pm 0,37^{\text {Aa }}$ & $0,00 \pm 0,00^{\mathrm{Aa}}$ & $0,00 \pm 0,00^{\mathrm{Aa}}$ & $0,00 \pm 0,00^{\mathrm{Aa}}$ \\
\hline $\mathrm{N}: \mathrm{L}$ & $3,44 \pm 2,24^{\mathrm{Aa}}$ & $0,70 \pm 0,44^{\mathrm{Ab}}$ & $0,66 \pm 0,33^{\mathrm{Ab}}$ & $0,32 \pm 0,20^{\mathrm{Ab}}$ & $0,26 \pm 0,16^{\mathrm{Ab}}$ & $0,20 \pm 0,15^{\mathrm{Ab}}$ & $0,16 \pm 0,08^{\mathrm{Ab}}$ & $0,25 \pm 0,18^{\mathrm{Ab}}$ \\
\hline $\mathrm{Fib}(\mathrm{mg} / \mathrm{dL})$ & $300,0 \pm 81,7^{\text {Aa }}$ & $290,0 \pm 99,4^{\mathrm{Aa}}$ & $410,0 \pm 128,7^{\text {Аа }}$ & $430,0 \pm 182,9^{\text {Аа }}$ & $300,0 \pm 81,7^{\mathrm{Aa}}$ & $440,0 \pm 96,6^{\mathrm{Aa}}$ & $360,0 \pm 183,8^{\mathrm{Aa}}$ & $370,0 \pm 156,7^{\mathrm{A}}$ \\
\hline & $56 \pm 0,63^{\mathrm{Aa}}$ & $7,07 \pm 0,48^{\mathrm{Abd}}$ & $6,78 \pm 0,38^{\mathrm{Abe}}$ & $6,54 \pm 0,43^{\text {Ace }}$ & $6,56 \pm 0,34^{\text {Ace }}$ & $6,71 \pm 0,29^{\text {Acde }}$ & $6,94 \pm 0,13^{\text {Abe }}$ & \\
\hline \multicolumn{9}{|c|}{ Aberdeen Angus (Red Angus) } \\
\hline Leuco $\left(\times 10^{3} / \mu \mathrm{L}\right)$ & $8,37 \pm 3,00^{\mathrm{Ab}}$ & $8,85 \pm 2,04^{\mathrm{Ab}}$ & $9,17 \pm 2,27^{\mathrm{Ab}}$ & $10,83 \pm 2,58^{\mathrm{Abd}}$ & $13,22 \pm 2,37^{\mathrm{Acd}}$ & $16,54 \pm 3,99^{\text {Aa }}$ & $13,30 \pm 4,34^{\mathrm{Acd}}$ & $15,03 \pm 3,59^{\text {Aac }}$ \\
\hline Bast $(\mathrm{x}$ & $0,06 \pm 0,11^{\mathrm{Aa}}$ & $0,00 \pm 0,02^{\mathrm{Ab}}$ & $0,00 \pm 0,00^{\mathrm{Ab}}$ & $0,00 \pm 0,00^{\mathrm{Ab}}$ & $0,00 \pm 0,00^{\mathrm{Ab}}$ & $0,02 \pm 0,07^{\mathrm{Ab}}$ & $0,00 \pm 0,00^{\mathrm{Ab}}$ & $0,00 \pm 0,00^{\mathrm{Ab}}$ \\
\hline $\operatorname{Seg}\left(x 10^{3} / \mu \mathrm{L}\right)$ & $5,06 \pm 1,91^{\mathrm{Aa}}$ & $3,09 \pm 1,44^{\mathrm{Ab}}$ & $2,55 \pm 1,39^{\mathrm{Ab}}$ & $2,09 \pm 1,23^{\mathrm{Ab}}$ & $2,21 \pm 1,56^{\mathrm{Ab}}$ & $2,60 \pm 1,51^{\mathrm{Ab}}$ & $1,77 \pm 0,85^{\mathrm{Ab}}$ & $2,59 \pm 1,85^{\mathrm{Ab}}$ \\
\hline $\operatorname{Linf}\left(x 10^{3} / \mu \mathrm{L}\right)$ & $2,98 \pm 1,23^{\mathrm{Ab}}$ & $5,53 \pm 1,55^{\mathrm{Ae}}$ & $6,29 \pm 2,47^{\mathrm{Ae}}$ & $8,43 \pm 2,11^{\mathrm{Ad}}$ & $10,51 \pm 1,30^{\mathrm{Ac}}$ & $13,45 \pm 3,10^{\mathrm{Aa}}$ & $11,12 \pm 3,79^{\mathrm{Ac}}$ & $12,09 \pm 4,03^{\mathrm{Aac}}$ \\
\hline Mono $\left(x 10^{3} / \mu \mathrm{L}\right)$ & $0,23 \pm 0,22^{\mathrm{Aa}}$ & $0,22 \pm 0,22^{\mathrm{Aa}}$ & $0,36 \pm 0,34^{\mathrm{Aa}}$ & $0,27 \pm 0,24^{\mathrm{Aa}}$ & $0,49 \pm 0,53^{\mathrm{Aa}}$ & $0,29 \pm 0,17^{\mathrm{Aa}}$ & $0,35 \pm 0,37^{\mathrm{Aa}}$ & $0,30 \pm 0,31^{\mathrm{Aa}}$ \\
\hline $\operatorname{Eosi}\left(\mathrm{x} 10^{3} / \mu \mathrm{L}\right)$ & $04 \pm 0,5$ & $0,00 \pm 0,00^{\mathrm{Ab}}$ & $0,05 \pm 0,13^{\mathrm{Ab}}$ & $0,04 \pm 0,07^{\mathrm{Ab}}$ & $0,01 \pm 0,03^{\mathrm{Bb}}$ & $0,18 \pm 0,21^{\mathrm{Aa}}$ & $0,06 \pm 0,10^{\mathrm{Ab}}$ & $0,05 \pm 0,14^{\mathrm{Ab}}$ \\
\hline Baso $\left(x 10^{3} / \mu \mathrm{L}\right)$ & $0,04 \pm 0,05^{\mathrm{Aa}}$ & $0,01 \pm 0,03^{\mathrm{Ab}}$ & $0,00 \pm 0,00^{\mathrm{Ab}}$ & $0,00 \pm 0,00^{\mathrm{Ab}}$ & $0,00 \pm 0,00^{\mathrm{Ab}}$ & $0,00 \pm 0,00^{\mathrm{Ab}}$ & $0,00 \pm 0,00^{\mathrm{Ab}}$ & $0,00 \pm 0,00^{\mathrm{Ab}}$ \\
\hline $\mathrm{N}: \mathrm{L}$ & $1,82 \pm 0,60^{\mathrm{Aa}}$ & $0,59 \pm 0,31^{\mathrm{Ab}}$ & $0,58 \pm 0,75^{\mathrm{Ab}}$ & $0,25 \pm 0,16^{\mathrm{Ab}}$ & $0,21 \pm 0,14^{\mathrm{Ab}}$ & $0,20 \pm 0,12^{\mathrm{Ab}}$ & $0,17 \pm 0,09^{\mathrm{Ab}}$ & $0,26 \pm 0,26^{\mathrm{Ab}}$ \\
\hline Fib (mg/dL) & $350,0 \pm 116,8^{\mathrm{Aa}}$ & $366,7 \pm 115,5^{\text {Aa }}$ & $383,3 \pm 169,7^{\text {Aa }}$ & $366,7 \pm 107,3^{\text {Aa }}$ & $375,0 \pm 96,5^{\mathrm{Aa}}$ & $300,0 \pm 127,9^{\mathrm{B}}$ & $333,3 \pm 187,5^{\mathrm{Aa}}$ & $358,3 \pm 192,9^{A}$ \\
\hline PTP (g/dL) & $8,05 \pm 1,00^{\mathrm{Aa}}$ & $6,88 \pm 0,58^{\mathrm{Abd}}$ & $6,38 \pm 0,49^{\mathrm{Bce}}$ & $6,13 \pm 0,31^{\mathrm{Bc}}$ & $6,31 \pm 0,44^{\text {Ace }}$ & $6,47 \pm 0,40^{\mathrm{Acd}}$ & $6,74 \pm 0,35^{\text {Abe }}$ & $7,13 \pm 0,25^{\mathrm{Ab}}$ \\
\hline
\end{tabular}

${ }^{\mathrm{A} P a r a}$ letras maiúsculas iguais não há diferença significativa $(P>0,05)$ entre os grupos. aPara letras minúsculas iguais não há diferença significativa $(P$ $>0,05)$ entre os momentos.

\section{DISCUSSÃO}

Durante o período neonatal a tendência ao aumento dos valores de eritrócitos, $\mathrm{Hb}$ e VG, observada no presente trabalho, para a raça Aberdeen Angus (Red Angus) e Crioula Lageana variedade mocha (exceto para a variável Hb) [Tabela 1], assemelha-se com observações realizadas em bezerros da raça Holandesa [19]. Entretanto, bezerros holandeses apresentaram diminuição nos valores destas variáveis a partir do nascimento $(>6 \mathrm{~h}$ ) até o terceiro e quarto dia para o número de eritrócitos e $\mathrm{Hb}$, e até o quinto e sexto dia para o VG. Estas variações foram atribuídas às baixas concentrações de oxigênio no ambiente placentário elevando as concentrações de $\mathrm{Hb}$ ao nascimento, diluição plasmática pela absorção do colostro e as baixas concentrações de ferro no recém-nascido, interferindo 
na eritropoiese. Optou-se no presente trabalho por realizar as colheitas somente após 24 h de vida (entre 24 e 36 h), impedindo assim, a observação dos valores do eritrograma antes da interferência do meio extrauterino. As colheitas não foram realizadas antes da mamada do colostro para evitar rejeição dos bezerros recém-nascidos pelas suas mães e pela dificuldade de manejo dos bovinos deste experimento, os quais eram criados em sistema extensivo. Valores de maior amplitude para as variáveis número de eritrócitos, VG e $\mathrm{Hb}$ no primeiro dia de vida (até $8 \mathrm{~h}$ ), com tendência a diminuição para o VG e $\mathrm{Hb}$ durante os primeiros 30 dias foram observados em bezerras da raça Holandesa [3]. Este resultado difere do encontrado no presente estudo, a partir dos dois dias (M1) até os 30 dias de vida (M3).

As flutuações com tendência a diminuição de seus valores para a variável VCM no período neonatal, em ambas as raças (Tabela 1), corroboram com observações realizadas por outros autores em bezerros da raça Holandesa [3,19]. Tendência a diminuição dos valores de HCM no período neonatal também foi observado em bezerros da raça Holandesa [19], semelhante ao encontrado no presente trabalho para a raça Aberdeen Angus (Red Angus). No entanto, para a raça Crioula Lageana variedade mocha os valores desta variável apresentaram diminuição seguido por aumento neste período. Concordando com observações de outros autores em outras raças, os valores de CHCM apresentaram pequenas oscilações durante o primeiro mês de vida $[3,4,24]$ (Tabela 1 ).

As variações observadas para as variáveis número de eritrócitos, VG, VGM e CHCM, para a raça Aberdeen Angus (Red Angus), a partir dos 90 dias de idade (M5) [Tabela 1], podem ser atribuídas a infecção por hemoparasitos intraeritrocitários do gênero Babesia spp. e Anaplasma sp., e consequente hemólise nesta faixa etária [14]. O aumento do valor da CHCM, o que não seria esperado neste caso, ocorreu provavelmente devido à diminuição proporcionalmente maior dos valores do VG em relação aos valores de hemoglobina [4].

A elevação dos valores referentes a contagem total de leucócitos e linfócitos acima do intervalo de referência para a espécie [14], na faixa etária dos 90 dias (M5) até 180 dias (M8), em ambas as raças (Tabela 2), pode ser explicado pela infestação por carrapatos observada neste período. Em regiões tropicais e subtropicais onde os bovinos são infestados por carrapatos [Rhipicephalus (Boophilus) microplus] e a tristeza pa- rasitária bovina é enzoótica, ocorre premunição natural dos bovinos contra o Babesia spp. e Anaplasma sp., determinando quadro de leucocitose por linfocitose [5]. Resultado semelhante foi em bezerros das raças Nelore e Limousin criados extensivamente na região norte do Paraná [7].

$\mathrm{O}$ aumento do número absoluto de linfócitos e consequente diminuição da relação $\mathrm{N}: \mathrm{L}$, observados no período neonatal (Tabela 2), estão em consonância com os encontrados por outros autores em outras raças $[1,4,6,8,12,20]$. No presente trabalho a inversão da relação N:L ocorreu aos 15 dias (M2), semelhante ao observado em bezerros das raças Nelore e Limousin [8]. O aumento do número de linfócitos ocorre por um possível efeito da redução da taxa de glicocorticóides endógenos após o parto [1]. A concentração do hormônio apresenta declínio nos primeiros dias de vida e continua a decair até aproximadamente 12 dias de vida, acompanhada pela diminuição da contagem de neutrófilos [11]. Além disso, imediatamente após o nascimento o número de linfócitos é baixo também devido ao desenvolvimento incompleto dos órgãos linfáticos [25].

O aumento significativo dos valores absolutos de eosinófilos na raça Aberdeen Angus (Red Angus), mesmo dentro do intervalo de referência para a espécie [14] (Tabela 2), pode ser atribuído ao período de infestação por carrapatos e consequente infecção por hemoparasitos. O aumento do número de eosinófilos pode estar presente em resposta à infecção por endoparasitos e infestações por ectoparasitos [27]. No entanto, este achado pode não ser observado em animais acometidos por estas enfermidades. Em uma avaliação hematológica em bezerros Nelore infectados experimentalmente com isolados de Babesia bigemina, foi observado aumento significativo nos valores absolutos de eosinófilos ao $23^{\circ}$ e $30^{\circ}$ dia pós-inoculação [17].

Não foram encontradas explicações referentes às diferenças observadas para o número de neutrófilos bastonetes no segundo dia de vida (M1) em ambas as raças (Tabela 2). Esta observação é difícil de ser analisada frente ao pequeno número de trabalhos que discutiram detalhadamente as variações destas células de acordo com a faixa etária [5]. Da mesma forma, a diferença observada para o número absoluto de basófilos, com relação a faixa etária, para a raça Aberdeen Angus (Red Angus), foi considerado um achado inespecífico. 
Os valores referentes a concentração de fibrinogênio plasmático permaneceram dentro do intervalo de referência para a espécie [15] durante o período experimental em ambas as raças (Tabela 2). No entanto, maiores valores foram encontrados para a raça Crioula Lageana variedade mocha quando comparado a raça Aberdeen Angus aos 120 dias de vida (M6). O aumento da concentração de fibrinogênio plasmático ocorre em resposta a processos inflamatórios e/ou infecciosos, sendo a onfalite e diarreia causas comuns em bezerros [22]. No entanto, estas alterações não foram observadas nos bezerros avaliados durante o período experimental no presente estudo.

Os maiores valores de PTP observados em ambas as raças no segundo dia de vida (M1) [Tabela 2] estão relacionados à ingestão do colostro e subsequente pico de absorção de imunoglobulinas [7]. A proteína total dosada por refratometria fornece uma estimativa indireta da quantidade de imunoglobulinas absorvidas devido à elevação da fração gamaglobulina e consequentemente da concentração total de proteínas [18]. Em estudo conduzido com bezerros das raças Nelore e Limousin foi observada alta correlação entre os valores de gamaglobulinas e PTP $(r=0,807)$ no segundo dia de vida (24-36 h) [7]. Sendo assim, com base nestas asserções, pode-se afirmar que houve adequada absorção de imunoglobulinas por meio do colostro nos animais avaliados em ambas as raças. A redução dos valores de PTP até os 60 dias de idade (M4), em ambas as raças, está relacionada à degradação e a combinação de imunoglobulinas com antígenos.
Por outro lado, o aumento gradativo dos valores de PTP, a partir dos 60 dias de idade (M4), está relacionado a síntese ativa de imunoglobulinas pelo sistema imune competente $[7,26]$.

\section{CONCLUSÃO}

As flutuações observadas nas variáveis do hemograma durante o período neonatal ocorreram de maneira semelhante às observadas em bezerros de outras raças. Alterações referentes à infecção e consequente premunição natural contra hemoparasitos devem ser consideradas durante a interpretação do hemograma de bovinos criados em regiões tropicais e subtropicais. Pela análise dos valores de PTP, ambas as raças apresentaram adequada transferência de imunidade passiva, e as flutuações desta variável ocorreram de maneira semelhante às descritas para bezerros de outras raças.

\section{MANUFACTURERS}

${ }^{1}$ Becton Dickinson \& Co. Curitiba, PR, Brazil. ${ }^{2}$ Laborclin Produtos para Laboratórios Ltda. Pinhais, PR, Brazil. ${ }^{3}$ ATAGO Co. Tokyo, Japan.

${ }^{4}$ MSD Saúde Animal. São Paulo, SP, Brazil.

Ethical approval. Samples used in this study were obtained with owners consent, and the procedures were approved by the Ethical Committee in Animal Experimentation of Universidade do Estado de Santa Catarina (No. 1.20.10).

Declaration of interest. None of the authors of this paper has a financial of personal relationship with other people or organizations that could inappropriately influence or bias the content of the paper.

\section{REFERENCES}

1 Adams R., Garry F.B., Aldridge B.M., Holland M.D. \& Odde K.G. 1992. Hematologic values in newborn beef calves. American Journal of Veterinary Research. 53(6): 944-950.

2 Assis-Brasil N.D., Pereira C.M., Hinnah F.L., Ladeira S.R.L., Sallis E.S.V., Grecco F.B. \& Schild A.L. 2013. Enfermidades diagnosticadas em bezerros na região sul do Rio Grande do Sul. Pesquisa Veterinária Brasileira. 33(4): 423-430.

3 Benesi F.J., Teixeira C.M.C., Lisboa J.A.N., Leal M.L.R., Birgel Júnior E.H., Bohland E. \& Mirandola R.M.S. 2012. Eritrograma de bezerras sadias da raça Holandesa no primeiro mês de vida. Pesquisa Veterinária Brasileira. 32(4): 357-360.

4 Biondo A.W., Lopes S.T.A., Kohayagawa A., Takahira R.K. \& Alencar N.X. 1998. Hemograma de bovinos (Bos indicus) sadios da raça Nelore no primeiro mês de vida, criados no estado de São Paulo. Ciência Rural. 28(2): 251-256.

5 Birgel junior E.H., D’Angelino J.L., Benesi F.J. \& Birgel E.H. 2001. Valores de referência do leucograma de bovinos da raça Jersey criados no Estado de São Paulo. Brazilian Journal of Veterinary Research Animal Science. 38(3): 136-141.

6 Costa J.N. 2000. Leucograma, metabolismo oxidativo dos neutrófilos, proteinograma e imunoglobulinas de bovinos da raça holandesa (Bos taurus). Influência do desenvolvimento etário e da suplementação com vitamina E (acetato de DL-alfa tocoferol). 209f. Botucatu, SP. Tese (Doutorado em Clínica Veterinária) - Programa de Pós-graduação em Clínica Veterinária, Universidade Estadual Paulista.

7 Costa M.C., Flaiban K.K.M.C., Coneglian M.M., Feitosa F.L.F., Balarin M.R.S. \& Lisbôa J.A.N. 2008. Transferência de imunidade passiva em bezerros das raças Nelore e Limousin e proteinograma sérico nos primeiros quatro meses de vida. Pesquisa Veterinária Brasileira. 28(9): 410-416. 
8 Costa M.C., Flaiban K.K.M.C., Coneglian M.M., Dognani R., Vettorato E.D., Balarin M.R.S. \& Lisbôa J.A.N. 2008. Metabolismo oxidativo dos neutrófilos de bezerros das raças Nelore e Limousin nos primeiros quatro meses de vida. Pesquisa Veterinária Brasileira. 28(9): 431-436.

9 Curi P.R. 1998. Metodologia e análise da pesquisa em ciências biológicas. 2.ed. Botucatu: Gráfica e Editora Tipomic, 263p.

10 Delfino J.L., Barbosa V.M., Gondim C.C., Oliveira P.M., Nasciutti N.R., Oliveira R.S.B.R., Tsuruta S.A., Mundim A.V. \& Saut J.P.E. 2014. Perfil bioquímico sérico de bezerros senepol nos primeiros 120 dias de idade. Semina: Ciência Agrárias. 35(3): 1341-1350.

11 Ebehart R.J. \& Patt J.A. 1971. Plasma cortisol concentrations in newborn calves. American Journal of Veterinary Research. 32(12): 1921-1927.

12 Fagliari J.J., Santana A.E., Lucas F.A., Campos Filho E. \& Curi P.R. 1998. Constituintes sangüíneos de bovinos recémnascidos das raças Nelore (Bos indicus) e Holandesa (Bos taurus) e de bubalinos (Bubalis bubalus) da raça Murrah. Arquivo Brasileiro de Medicina Veterinária e Zootecnia. 50(5): 253-262.

13 Gonçalves R.C., Peixoto A.P.C., Kuchembuck M.R., Chiacchio S.B., Kohayagawa A. \& Castro A.A.P. 1993. Doenças de bezerros. Pneumonia. Aspectos clínicos e epidemiológicos na região de Botucatu. In: Resumos do XI Congresso Internacional de Medicina Veterinária em Língua Portuguesa (Salvador, Brasil). p.289.

14 Jain N.C. 1993. Essentials of veterinary hematology. Philadelphia: Lea \& Febiger, 417p.

15 Kaneko J.J., Harvey J.W. \& Bruss M.L. 2008. Clinical biochemistry of domestic animals. 6th edn. San Diego: Academic Press, 928p.

16 Machado Neto R., Packer I.V., Bonilha L.M., Figueredo L.A., Razzok A.G. \& Candido J.G. 1997. Concentração de IgG sérica em bezerros das raças Nelore, Guzerá, Gir e Caracu. Efeito sobre o crescimento e a mortalidade até a desmama. Revista Brasileira de Zootecnia. 26: 920-923.

17 Mendonça C.L., Vieira D., Kohayahawa A., Schenk M.A.M., Madruga C.R. \& Afonso J.A.B. 2003. Avaliação clínica e hematológica em bezerros Nelore infectados experimentalmente com isolados de Babesia bigemina das regiões Sudeste, Nordeste e Norte do Brasil. Pesquisa Veterinária Brasileira. 23(2): 52-60.

18 Naylor J.M., Kronfeld D.S., Bech-Nielsen S. \& Bartholomew R.C. 1977. Plasma total protein measurement for prediction of disease and mortality in calves. Journal of the American Veterinary Medical Association. 171(7): 635-638.

19 Novo S.M.F., Freitas R.L., Silva C.P.C., Baldacim V.A.P., Baccili C.C., Reis J.F., Hagiwara M.K. \& Gomes V. 2015. Hematological adaptation in Holstein calves during the neonatal period. Brazilian Journal of Veterinary Research and Animal Science. 52(3): 212-216.

20 Peixoto A.P.C., Costa J.N., Kohayagawa A., Takahira R.K. \& Saito M.E. 2002. Hemograma e metabolismo oxidativo dos neutrófilos de bovinos da raça Holandesa preta e branca: influência dos fatores etários. Revista Brasileira de Saúde Produção Animal. 3(1): 16-20.

21 Radostits O.M., Gay C.C., Blood D.C. \& Hinchcliff K.W. 2002. Clínica Veterinária: Um tratado de doenças dos bovinos, ovinos, suínos, caprinos e equinos. 9.ed. Rio de Janeiro: Guanabara Koogan, 1737p.

22 Rengifo S.A., Silva R.A., Botteon R.C.C.M. \& Botteon P.T.L. 2010. Hemograma e bioquímica sérica auxiliar em bezerros mestiços neonatos e ocorrência de enfermidades. Arquivo Brasileiro de Medicina Veterinária e Zootecnia. 62(4): 993-997.

23 Rocha T.G., Franciosi C., Nociti R.P., Nogueira C.A.S. \& Fagliari J.J. 2010. Hemograma e proteínas do soro sanguíneo de bezerros Canchim-Nelore e da raça Holandesa nos primeiros 30 dias de vida. Arquivo Brasileiro de Medicina Veterinária e Zootecnia. 62(5): 1250-1254.

24 Rocha T.G., Nociti R.P., Sampaio A.A.M. \& Fagliari J.J. 2013. Hemograma e proteínas de fase aguda de bezerros sadios do nascimento aos 30 dias de idade. Pesquisa Veterinária Brasileira. 33(1): 25-31.

25 Soliman M.K. \& Zaki K. 1967. Blood picture of Friesian heifers from birth till maturity. Zentralblatt für Veterinarmedizin, Reihe A. 13: 467-473.

26 Teixeira W.T., Fonteque G.V., Ramos A.F., Mariante A.S., Egito A.A., Martins V.V., Saito M.E. \& Fonteque J.H. 2012. Transfer of passive immunity and serum proteinogram in the first six months of life of Criollo Lageano and Black and White Holstein calves. Pesquisa Veterinária Brasileira. 32(10): 980-986.

27 Tornquist S.J. \& Rigas J. 2010. Interpretation of ruminant leukocyte responses. In: Weiss D.J. \& Wardrop K.J. (Eds). Schalm's veterinary hematology. 6th edn. Ames: Wiley-Blackwell, pp.307-313. 